\title{
ON PROOF AND PROGRESS IN MATHEMATICS
}

\author{
WILLIAM P. THURSTON
}

This essay on the nature of proof and progress in mathematics was stimulated by the article of Jaffe and Quinn, "Theoretical Mathematics: Toward a cultural synthesis of mathematics and theoretical physics". Their article raises interesting issues that mathematicians should pay more attention to, but it also perpetuates some widely held beliefs and attitudes that need to be questioned and examined.

The article had one paragraph portraying some of my work in a way that diverges from my experience, and it also diverges from the observations of people in the field whom I've discussed it with as a reality check.

After some reflection, it seemed to me that what Jaffe and Quinn wrote was an example of the phenomenon that people see what they are tuned to see. Their portrayal of my work resulted from projecting the sociology of mathematics onto a one-dimensional scale (speculation versus rigor) that ignores many basic phenomena.

Responses to the Jaffe-Quinn article have been invited from a number of mathematicians, and I expect it to receive plenty of specific analysis and criticism from others. Therefore, I will concentrate in this essay on the positive rather than on the contranegative. I will describe my view of the process of mathematics, referring only occasionally to Jaffe and Quinn by way of comparison.

In attempting to peel back layers of assumptions, it is important to try to begin with the right questions:

\section{What IS IT THAT MATHEMATICIANS ACCOMPLiSH?}

There are many issues buried in this question, which I have tried to phrase in a way that does not presuppose the nature of the answer.

It would not be good to start, for example, with the question

How do mathematicians prove theorems?

This question introduces an interesting topic, but to start with it would be to project two hidden assumptions:

(1) that there is uniform, objective and firmly established theory and practice of mathematical proof, and

(2) that progress made by mathematicians consists of proving theorems.

It is worthwhile to examine these hypotheses, rather than to accept them as obvious and proceed from there.

Received by the editors October 26, 1993.

1991 Mathematics Subject Classification. Primary 01 A80. 
The question is not even

How do mathematicians make progress in mathematics?

Rather, as a more explicit (and leading) form of the question, I prefer

How do mathematicians advance human understanding of mathematics?

This question brings to the fore something that is fundamental and pervasive: that what we are doing is finding ways for people to understand and think about mathematics.

The rapid advance of computers has helped dramatize this point, because computers and people are very different. For instance, when Appel and Haken completed a proof of the 4-color map theorem using a massive automatic computation, it evoked much controversy. I interpret the controversy as having little to do with doubt people had as to the veracity of the theorem or the correctness of the proof. Rather, it reflected a continuing desire for human understanding of a proof, in addition to knowledge that the theorem is true.

On a more everyday level, it is common for people first starting to grapple with computers to make large-scale computations of things they might have done on a smaller scale by hand. They might print out a table of the first 10,000 primes, only to find that their printout isn't something they really wanted after all. They discover by this kind of experience that what they really want is usually not some collection of "answers"-what they want is understanding.

It may sound almost circular to say that what mathematicians are accomplishing is to advance human understanding of mathematics. I will not try to resolve this by discussing what mathematics is, because it would take us far afield. Mathematicians generally feel that they know what mathematics is, but find it difficult to give a good direct definition. It is interesting to try. For me, "the theory of formal patterns" has come the closest, but to discuss this would be a whole essay in itself.

Could the difficulty in giving a good direct definition of mathematics be an essential one, indicating that mathematics has an essential recursive quality? Along these lines we might say that mathematics is the smallest subject satisfying the following:

- Mathematics includes the natural numbers and plane and solid geometry.

- Mathematics is that which mathematicians study.

- Mathematicians are those humans who advance human understanding of mathematics.

In other words, as mathematics advances, we incorporate it into our thinking. As our thinking becomes more sophisticated, we generate new mathematical concepts and new mathematical structures: the subject matter of mathematics changes to reflect how we think.

If what we are doing is constructing better ways of thinking, then psychological and social dimensions are essential to a good model for mathematical progress. These dimensions are absent from the popular model. In caricature, the popular model holds that 
D. mathematicians start from a few basic mathematical structures and a collection of axioms "given" about these structures, that

T. there are various important questions to be answered about these structures that can be stated as formal mathematical propositions, and

P. the task of the mathematician is to seek a deductive pathway from the axioms to the propositions or to their denials.

We might call this the definition-theorem-proof (DTP) model of mathematics.

A clear difficulty with the DTP model is that it doesn't explain the source of the questions. Jaffe and Quinn discuss speculation (which they inappropriately label "theoretical mathematics") as an important additional ingredient. Speculation consists of making conjectures, raising questions, and making intelligent guesses and heuristic arguments about what is probably true.

Jaffe and Quinn's DSTP model still fails to address some basic issues. We are not trying to meet some abstract production quota of definitions, theorems and proofs. The measure of our success is whether what we do enables people to understand and think more clearly and effectively about mathematics.

Therefore, we need to ask ourselves:

\section{HoW DO PEOPLE UNDERSTAND MATHEMATICS?}

This is a very hard question. Understanding is an individual and internal matter that is hard to be fully aware of, hard to understand and often hard to communicate. We can only touch on it lightly here.

People have very different ways of understanding particular pieces of mathematics. To illustrate this, it is best to take an example that practicing mathematicians understand in multiple ways, but that we see our students struggling with. The derivative of a function fits well. The derivative can be thought of as:

(1) Infinitesimal: the ratio of the infinitesimal change in the value of a function to the infinitesimal change in a function.

(2) Symbolic: the derivative of $x^{n}$ is $n x^{n-1}$, the derivative of $\sin (x)$ is $\cos (x)$, the derivative of $f \circ g$ is $f^{\prime} \circ g * g^{\prime}$, etc.

(3) Logical: $f^{\prime}(x)=d$ if and only if for every $\epsilon$ there is a $\delta$ such that when $0<|\Delta x|<\delta$,

$$
\left|\frac{f(x+\Delta x)-f(x)}{\Delta x}-d\right|<\delta .
$$

(4) Geometric: the derivative is the slope of a line tangent to the graph of the function, if the graph has a tangent.

(5) Rate: the instantaneous speed of $f(t)$, when $t$ is time.

(6) Approximation: The derivative of a function is the best linear approximation to the function near a point.

(7) Microscopic: The derivative of a function is the limit of what you get by looking at it under a microscope of higher and higher power.

This is a list of different ways of thinking about or conceiving of the derivative, rather than a list of different logical definitions. Unless great efforts are made to maintain the tone and flavor of the original human insights, the differences 
start to evaporate as soon as the mental concepts are translated into precise, formal and explicit definitions.

I can remember absorbing each of these concepts as something new and interesting, and spending a good deal of mental time and effort digesting and practicing with each, reconciling it with the others. I also remember coming back to revisit these different concepts later with added meaning and understanding.

The list continues; there is no reason for it ever to stop. A sample entry further down the list may help illustrate this. We may think we know all there is to say about a certain subject, but new insights are around the corner. Furthermore, one person's clear mental image is another person's intimidation:

37. The derivative of a real-valued function $f$ in a domain $D$ is the Lagrangian section of the cotangent bundle $T^{*}(D)$ that gives the connection form for the unique flat connection on the trivial $\mathbf{R}$-bundle $D \times \mathbf{R}$ for which the graph of $f$ is parallel.

These differences are not just a curiosity. Human thinking and understanding do not work on a single track, like a computer with a single central processing unit. Our brains and minds seem to be organized into a variety of separate, powerful facilities. These facilities work together loosely, "talking" to each other at high levels rather than at low levels of organization.

Here are some major divisions that are important for mathematical thinking:

(1) Human language. We have powerful special-purpose facilities for speaking and understanding human language, which also tie in to reading and writing. Our linguistic facility is an important tool for thinking, not just for communication. A crude example is the quadratic formula which people may remember as a little chant, "ex equals minus bee plus or minus the square root of bee squared minus four ay see all over two ay." The mathematical language of symbols is closely tied to our human language facility. The fragment of mathematical symbolese available to most calculus students has only one verb, “=”. That's why students use it when they're in need of a verb. Almost anyone who has taught calculus in the U.S. has seen students instinctively write " $x^{3}=3 x^{2}$ " and the like.

(2) Vision, spatial sense, kinesthetic (motion) sense. People have very powerful facilities for taking in information visually or kinesthetically, and thinking with their spatial sense. On the other hand, they do not have a very good built-in facility for inverse vision, that is, turning an internal spatial understanding back into a two-dimensional image. Consequently, mathematicians usually have fewer and poorer figures in their papers and books than in their heads.

An interesting phenomenon in spatial thinking is that scale makes a big difference. We can think about little objects in our hands, or we can think of bigger human-sized structures that we scan, or we can think of spatial structures that encompass us and that we move around in. We tend to think more effectively with spatial imagery on a larger scale: it's as if our brains take larger things more seriously and can devote more resources to them.

(3) Logic and deduction. We have some built-in ways of reasoning and 
putting things together associated with how we make logical deductions: cause and effect (related to implication), contradiction or negation, etc.

Mathematicians apparently don't generally rely on the formal rules of deduction as they are thinking. Rather, they hold a fair bit of logical structure of a proof in their heads, breaking proofs into intermediate results so that they don't have to hold too much logic at once. In fact, it is common for excellent mathematicians not even to know the standard formal usage of quantifiers (for all and there exists), yet all mathematicians certainly perform the reasoning that they encode.

It's interesting that although "or", "and" and "implies" have identical formal usage, we think of "or" and "and" as conjunctions and "implies" as a verb.

(4) Intuition, association, metaphor. People have amazing facilities for sensing something without knowing where it comes from (intuition); for sensing that some phenomenon or situation or object is like something else (association); and for building and testing connections and comparisons, holding two things in mind at the same time (metaphor). These facilities are quite important for mathematics. Personally, I put a lot of effort into "listening" to my intuitions and associations, and building them into metaphors and connections. This involves a kind of simultaneous quieting and focusing of my mind. Words, logic, and detailed pictures rattling around can inhibit intuitions and associations.

(5) Stimulus-response. This is often emphasized in schools; for instance, if you see $3927 \times 253$, you write one number above the other and draw a line underneath, etc. This is also important for research mathematics: seeing a diagram of a knot, I might write down a presentation for the fundamental group of its complement by a procedure that is similar in feel to the multiplication algorithm.

(6) Process and time. We have a facility for thinking about processes or sequences of actions that can often be used to good effect in mathematical reasoning. One way to think of a function is as an action, a process, that takes the domain to the range. This is particularly valuable when composing functions. Another use of this facility is in remembering proofs: people often remember a proof as a process consisting of several steps. In topology, the notion of a homotopy is most often thought of as a process taking time. Mathematically, time is no different from one more spatial dimension, but since humans interact with it in a quite different way, it is psychologically very different.

\section{How is MATHEMATICAL UNDERSTANDING COMMUNICATED?}

The transfer of understanding from one person to another is not automatic. It is hard and tricky. Therefore, to analyze human understanding of mathematics, it is important to consider who understands what, and when.

Mathematicians have developed habits of communication that are often dysfunctional. Organizers of colloquium talks everywhere exhort speakers to explain things in elementary terms. Nonetheless, most of the audience at an average colloquium talk gets little of value from it. Perhaps they are lost within the first 5 minutes, yet sit silently through the remaining 55 minutes. Or perhaps they quickly lose interest because the speaker plunges into technical details 
without presenting any reason to investigate them. At the end of the talk, the few mathematicians who are close to the field of the speaker ask a question or two to avoid embarrassment.

This pattern is similar to what often holds in classrooms, where we go through the motions of saying for the record what we think the students "ought" to learn, while the students are trying to grapple with the more fundamental issues of learning our language and guessing at our mental models. Books compensate by giving samples of how to solve every type of homework problem. Professors compensate by giving homework and tests that are much easier than the material "covered" in the course, and then grading the homework and tests on a scale that requires little understanding. We assume that the problem is with the students rather than with communication: that the students either just don't have what it takes, or else just don't care.

Outsiders are amazed at this phenomenon, but within the mathematical community, we dismiss it with shrugs.

Much of the difficulty has to do with the language and culture of mathematics, which is divided into subfields. Basic concepts used every day within one subfield are often foreign to another subfield. Mathematicians give up on trying to understand the basic concepts even from neighboring subfields, unless they were clued in as graduate students.

In contrast, communication works very well within the subfields of mathematics. Within a subfield, people develop a body of common knowledge and known techniques. By informal contact, people learn to understand and copy each other's ways of thinking, so that ideas can be explained clearly and easily.

Mathematical knowledge can be transmitted amazingly fast within a subfield. When a significant theorem is proved, it often (but not always) happens that the solution can be communicated in a matter of minutes from one person to another within the subfield. The same proof would be communicated and generally understood in an hour talk to members of the subfield. It would be the subject of a 15- or 20-page paper, which could be read and understood in a few hours or perhaps days by members of the subfield.

Why is there such a big expansion from the informal discussion to the talk to the paper? One-on-one, people use wide channels of communication that go far beyond formal mathematical language. They use gestures, they draw pictures and diagrams, they make sound effects and use body language. Communication is more likely to be two-way, so that people can concentrate on what needs the most attention. With these channels of communication, they are in a much better position to convey what's going on, not just in their logical and linguistic facilities, but in their other mental facilities as well.

In talks, people are more inhibited and more formal. Mathematical audiences are often not very good at asking the questions that are on most people's minds, and speakers often have an unrealistic preset outline that inhibits them from addressing questions even when they are asked.

In papers, people are still more formal. Writers translate their ideas into symbols and logic, and readers try to translate back.

Why is there such a discrepancy between communication within a subfield and communication outside of subfields, not to mention communication outside mathematics? 
Mathematics in some sense has a common language: a language of symbols, technical definitions, computations, and logic. This language efficiently conveys some, but not all, modes of mathematical thinking. Mathematicians learn to translate certain things almost unconsciously from one mental mode to the other, so that some statements quickly become clear. Different mathematicians study papers in different ways, but when I read a mathematical paper in a field in which I'm conversant, I concentrate on the thoughts that are between the lines. I might look over several paragraphs or strings of equations and think to myself "Oh yeah, they're putting in enough rigamarole to carry such-andsuch idea." When the idea is clear, the formal setup is usually unnecessary and redundant-I often feel that I could write it out myself more easily than figuring out what the authors actually wrote. It's like a new toaster that comes with a 16-page manual. If you already understand toasters and if the toaster looks like previous toasters you've encountered, you might just plug it in and see if it works, rather than first reading all the details in the manual.

People familiar with ways of doing things in a subfield recognize various patterns of statements or formulas as idioms or circumlocution for certain concepts or mental images. But to people not already familiar with what's going on the same patterns are not very illuminating; they are often even misleading. The language is not alive except to those who use it.

I'd like to make an important remark here: there are some mathematicians who are conversant with the ways of thinking in more than one subfield, sometimes in quite a number of subfields. Some mathematicians learn the jargon of several subfields as graduate students, some people are just quick at picking up foreign mathematical language and culture, and some people are in mathematical centers where they are exposed to many subfields. People who are comfortable in more than one subfield can often have a very positive influence, serving as bridges, and helping different groups of mathematicians learn from each other. But people knowledgeable in multiple fields can also have a negative effect, by intimidating others, and by helping to validate and maintain the whole system of generally poor communication. For example, one effect often takes place during colloquium talks, where one or two widely knowledgeable people sitting in the front row may serve as the speaker's mental guide to the audience.

There is another effect caused by the big differences between how we think about mathematics and how we write it. A group of mathematicians interacting with each other can keep a collection of mathematical ideas alive for a period of years, even though the recorded version of their mathematical work differs from their actual thinking, having much greater emphasis on language, symbols, logic and formalism. But as new batches of mathematicians learn about the subject they tend to interpret what they read and hear more literally, so that the more easily recorded and communicated formalism and machinery tend to gradually take over from other modes of thinking.

There are two counters to this trend, so that mathematics does not become entirely mired down in formalism. First, younger generations of mathematicians are continually discovering and rediscovering insights on their own, thus reinjecting diverse modes of human thought into mathematics. 
Second, mathematicians sometimes invent names and hit on unifying definitions that replace technical circumlocutions and give good handles for insights. Names like "group" to replace "a system of substitutions satisfying ... ", and "manifold" to replace

We can't give coordinates to parametrize all the solutions to our equations simultaneously, but in the neighborhood of any particular solution we can introduce coordinates

$$
\begin{aligned}
& \left(f_{1}\left(u_{1}, u_{2}, u_{3}\right), f_{2}\left(u_{1}, u_{2}, u_{3}\right), f_{3}\left(u_{1}, u_{2}, u_{3}\right), f_{4}\left(u_{1}, u_{2}, u_{3}\right),\right. \\
& \left.f_{5}\left(u_{1}, u_{2}, u_{3}\right)\right)
\end{aligned}
$$

where at least one of the ten determinants

...[ten $3 \times 3$ determinants of matrices of partial derivatives]...

is not zero

may or may not have represented advances in insight among experts, but they greatly facilitate the communication of insights.

We mathematicians need to put far greater effort into communicating mathematical ideas. To accomplish this, we need to pay much more attention to communicating not just our definitions, theorems, and proofs, but also our ways of thinking. We need to appreciate the value of different ways of thinking about the same mathematical structure.

We need to focus far more energy on understanding and explaining the basic mental infrastructure of mathematics-with consequently less energy on the most recent results. This entails developing mathematical language that is effective for the radical purpose of conveying ideas to people who don't already know them.

Part of this communication is through proofs.

\section{WHAT IS A PROOF?}

When I started as a graduate student at Berkeley, I had trouble imagining how I could "prove" a new and interesting mathematical theorem. I didn't really understand what a "proof" was.

By going to seminars, reading papers, and talking to other graduate students, I gradually began to catch on. Within any field, there are certain theorems and certain techniques that are generally known and generally accepted. When you write a paper, you refer to these without proof. You look at other papers in the field, and you see what facts they quote without proof, and what they cite in their bibliography. You learn from other people some idea of the proofs. Then you're free to quote the same theorem and cite the same citations. You don't necessarily have to read the full papers or books that are in your bibliography. Many of the things that are generally known are things for which there may be no known written source. As long as people in the field are comfortable that the idea works, it doesn't need to have a formal written source. 
At first I was highly suspicious of this process. I would doubt whether a certain idea was really established. But I found that I could ask people, and they could produce explanations and proofs, or else refer me to other people or to written sources that would give explanations and proofs. There were published theorems that were generally known to be false, or where the proofs were generally known to be incomplete. Mathematical knowledge and understanding were embedded in the minds and in the social fabric of the community of people thinking about a particular topic. This knowledge was supported by written documents, but the written documents were not really primary.

I think this pattern varies quite a bit from field to field. I was interested in geometric areas of mathematics, where it is often pretty hard to have a document that reflects well the way people actually think. In more algebraic or symbolic fields, this is not necessarily so, and I have the impression that in some areas documents are much closer to carrying the life of the field. But in any field, there is a strong social standard of validity and truth. Andrew Wiles's proof of Fermat's Last Theorem is a good illustration of this, in a field which is very algebraic. The experts quickly came to believe that his proof was basically correct on the basis of high-level ideas, long before details could be checked. This proof will receive a great deal of scrutiny and checking compared to most mathematical proofs; but no matter how the process of verification plays out, it helps illustrate how mathematics evolves by rather organic psychological and social processes.

When people are doing mathematics, the flow of ideas and the social standard of validity is much more reliable than formal documents. People are usually not very good in checking formal correctness of proofs, but they are quite good at detecting potential weaknesses or flaws in proofs.

To avoid misinterpretation, I'd like to emphasize two things I am not saying. First, I am not advocating any weakening of our community standard of proof; I am trying to describe how the process really works. Careful proofs that will stand up to scrutiny are very important. I think the process of proof on the whole works pretty well in the mathematical community. The kind of change I would advocate is that mathematicians take more care with their proofs, making them really clear and as simple as possible so that if any weakness is present it will be easy to detect. Second, I am not criticizing the mathematical study of formal proofs, nor am I criticizing people who put energy into making mathematical arguments more explicit and more formal. These are both useful activities that shed new insights on mathematics.

I have spent a fair amount of effort during periods of my career exploring mathematical questions by computer. In view of that experience, I was astonished to see the statement of Jaffe and Quinn that mathematics is extremely slow and arduous, and that it is arguably the most disciplined of all human activities. The standard of correctness and completeness necessary to get a computer program to work at all is a couple of orders of magnitude higher than the mathematical community's standard of valid proofs. Nonetheless, large computer programs, even when they have been very carefully written and very carefully tested, always seem to have bugs. 
I think that mathematics is one of the most intellectually gratifying of human activities. Because we have a high standard for clear and convincing thinking and because we place a high value on listening to and trying to understand each other, we don't engage in interminable arguments and endless redoing of our mathematics. We are prepared to be convinced by others. Intellectually, mathematics moves very quickly. Entire mathematical landscapes change and change again in amazing ways during a single career.

When one considers how hard it is to write a computer program even approaching the intellectual scope of a good mathematical paper, and how much greater time and effort have to be put into it to make it "almost" formally correct, it is preposterous to claim that mathematics as we practice it is anywhere near formally correct.

Mathematics as we practice it is much more formally complete and precise than other sciences, but it is much less formally complete and precise for its content than computer programs. The difference has to do not just with the amount of effort: the kind of effort is qualitatively different. In large computer programs, a tremendous proportion of effort must be spent on myriad compatibility issues: making sure that all definitions are consistent, developing "good" data structures that have useful but not cumbersome generality, deciding on the "right" generality for functions, etc. The proportion of energy spent on the working part of a large program, as distinguished from the bookkeeping part, is surprisingly small. Because of compatibility issues that almost inevitably escalate out of hand because the "right" definitions change as generality and functionality are added, computer programs usually need to be rewritten frequently, often from scratch.

A very similar kind of effort would have to go into mathematics to make it formally correct and complete. It is not that formal correctness is prohibitively difficult on a small scale-it's that there are many possible choices of formalization on small scales that translate to huge numbers of interdependent choices in the large. It is quite hard to make these choices compatible; to do so would certainly entail going back and rewriting from scratch all old mathematical papers whose results we depend on. It is also quite hard to come up with good technical choices for formal definitions that will be valid in the variety of ways that mathematicians want to use them and that will anticipate future extensions of mathematics. If we were to continue to cooperate, much of our time would be spent with international standards commissions to establish uniform definitions and resolve huge controversies.

Mathematicians can and do fill in gaps, correct errors, and supply more detail and more careful scholarship when they are called on or motivated to do so. Our system is quite good at producing reliable theorems that can be solidly backed up. It's just that the reliability does not primarily come from mathematicians formally checking formal arguments; it comes from mathematicians thinking carefully and critically about mathematical ideas.

On the most fundamental level, the foundations of mathematics are much shakier than the mathematics that we do. Most mathematicians adhere to foundational principles that are known to be polite fictions. For example, it is a theorem that there does not exist any way to ever actually construct or even de- 
fine a well-ordering of the real numbers. There is considerable evidence (but no proof) that we can get away with these polite fictions without being caught out, but that doesn't make them right. Set theorists construct many alternate and mutually contradictory "mathematical universes" such that if one is consistent, the others are too. This leaves very little confidence that one or the other is the right choice or the natural choice. Gödel's incompleteness theorem implies that there can be no formal system that is consistent, yet powerful enough to serve as a basis for all of the mathematics that we do.

In contrast to humans, computers are good at performing formal processes. There are people working hard on the project of actually formalizing parts of mathematics by computer, with actual formally correct formal deductions. I think this is a very big but very worthwhile project, and I am confident that we will learn a lot from it. The process will help simplify and clarify mathematics. In not too many years, I expect that we will have interactive computer programs that can help people compile significant chunks of formally complete and correct mathematics (based on a few perhaps shaky but at least explicit assumptions), and that they will become part of the standard mathematician's working environment.

However, we should recognize that the humanly understandable and humanly checkable proofs that we actually do are what is most important to us, and that they are quite different from formal proofs. For the present, formal proofs are out of reach and mostly irrelevant: we have good human processes for checking mathematical validity.

\section{What Motivates PEOPle to Do MATHEMATICS?}

There is a real joy in doing mathematics, in learning ways of thinking that explain and organize and simplify. One can feel this joy discovering new mathematics, rediscovering old mathematics, learning a way of thinking from a person or text, or finding a new way to explain or to view an old mathematical structure.

This inner motivation might lead us to think that we do mathematics solely for its own sake. That's not true: the social setting is extremely important. We are inspired by other people, we seek appreciation by other people, and we like to help other people solve their mathematical problems. What we enjoy changes in response to other people. Social interaction occurs through face-to-face meetings. It also occurs through written and electronic correspondence, preprints, and journal articles. One effect of this highly social system of mathematics is the tendency of mathematicians to follow fads. For the purpose of producing new mathematical theorems this is probably not very efficient: we'd seem to be better off having mathematicians cover the intellectual field much more evenly. But most mathematicians don't like to be lonely, and they have trouble staying excited about a subject, even if they are personally making progress, unless they have colleagues who share their excitement.

In addition to our inner motivation and our informal social motivation for doing mathematics, we are driven by considerations of economics and status. Mathematicians, like other academics, do a lot of judging and being judged. Starting with grades, and continuing through letters of recommendation, hiring decisions, promotion decisions, referees reports, invitations to speak, prizes, ... we are involved in many ratings, in a fiercely competitive system. 
Jaffe and Quinn analyze the motivation to do mathematics in terms of a common currency that many mathematicians believe in: credit for theorems.

I think that our strong communal emphasis on theorem-credits has a negative effect on mathematical progress. If what we are accomplishing is advancing human understanding of mathematics, then we would be much better off recognizing and valuing a far broader range of activity. The people who see the way to proving theorems are doing it in the context of a mathematical community; they are not doing it on their own. They depend on understanding of mathematics that they glean from other mathematicians. Once a theorem has been proven, the mathematical community depends on the social network to distribute the ideas to people who might use them further-the print medium is far too obscure and cumbersome.

Even if one takes the narrow view that what we are producing is theorems, the team is important. Soccer can serve as a metaphor. There might only be one or two goals during a soccer game, made by one or two persons. That does not mean that the efforts of all the others are wasted. We do not judge players on a soccer team only by whether they personally make a goal; we judge the team by its function as a team.

In mathematics, it often happens that a group of mathematicians advances with a certain collection of ideas. There are theorems in the path of these advances that will almost inevitably be proven by one person or another. Sometimes the group of mathematicians can even anticipate what these theorems are likely to be. It is much harder to predict who will actually prove the theorem, although there are usually a few "point people" who are more likely to score. However, they are in a position to prove those theorems because of the collective efforts of the team. The team has a further function, in absorbing and making use of the theorems once they are proven. Even if one person could prove all the theorems in the path single-handedly, they are wasted if nobody else learns them.

There is an interesting phenomenon concerning the "point" people. It regularly happens that someone who was in the middle of a pack proves a theorem that receives wide recognition as being significant. Their status in the community-their pecking order-rises immediately and dramatically. When this happens, they usually become much more productive as a center of ideas and a source of theorems. Why? First, there is a large increase in self-esteem, and an accompanying increase in productivity. Second, when their status increases, people are more in the center of the network of ideas-others take them more seriously. Finally and perhaps most importantly, a mathematical breakthrough usually represents a new way of thinking, and effective ways of thinking can usually be applied in more than one situation.

This phenomenon convinces me that the entire mathematical community would become much more productive if we open our eyes to the real values in what we are doing. Jaffe and Quinn propose a system of recognized roles divided into "speculation" and "proving". Such a division only perpetuates the myth that our progress is measured in units of standard theorems deduced. This is a bit like the fallacy of the person who makes a printout of the first 10,000 primes. What we are producing is human understanding. We have many different ways to understand and many different processes that contribute 
to our understanding. We will be more satisfied, more productive and happier if we recognize and focus on this.

\section{SOME PERSONAL EXPERIENCES}

Since this essay grew out of reflection on the misfit between my experiences and the description of Jaffe and Quinn's, I will discuss two personal experiences, including the one they alluded to.

I feel some awkwardness in this, because I do have regrets about aspects of my career: if I were to do things over again with the benefit of my present insights about myself and about the process of mathematics, there is a lot that I would hope to do differently. I hope that by describing these experiences rather openly as I remember and understand them, I can help others understand the process better and learn in advance.

First I will discuss briefly the theory of foliations, which was my first subject, starting when I was a graduate student. (It doesn't matter here whether you know what foliations are.)

At that time, foliations had become a big center of attention among geometric topologists, dynamical systems people, and differential geometers. I fairly rapidly proved some dramatic theorems. I proved a classification theorem for foliations, giving a necessary and sufficient condition for a manifold to admit a foliation. I proved a number of other significant theorems. I wrote respectable papers and published at least the most important theorems. It was hard to find the time to write to keep up with what I could prove, and I built up a backlog.

An interesting phenomenon occurred. Within a couple of years, a dramatic evacuation of the field started to take place. I heard from a number of mathematicians that they were giving or receiving advice not to go into foliationsthey were saying that Thurston was cleaning it out. People told me (not as a complaint, but as a compliment) that I was killing the field. Graduate students stopped studying foliations, and fairly soon, I turned to other interests as well.

I do not think that the evacuation occurred because the territory was intellectually exhausted-there were (and still are) many interesting questions that remain and that are probably approachable. Since those years, there have been interesting developments carried out by the few people who stayed in the field or who entered the field, and there have also been important developments in neighboring areas that I think would have been much accelerated had mathematicians continued to pursue foliation theory vigorously.

Today, I think there are few mathematicians who understand anything approaching the state of the art of foliations as it lived at that time, although there are some parts of the theory of foliations, including developments since that time, that are still thriving.

I believe that two ecological effects were much more important in putting a damper on the subject than any exhaustion of intellectual resources that occurred.

First, the results I proved (as well as some important results of other people) were documented in a conventional, formidable mathematician's style. They depended heavily on readers who shared certain background and certain insights. The theory of foliations was a young, opportunistic subfield, and the background 
was not standardized. I did not hesitate to draw on any of the mathematics I had learned from others. The papers I wrote did not (and could not) spend much time explaining the background culture. They documented top-level reasoning and conclusions that I often had achieved after much reflection and effort. I also threw out prize cryptic tidbits of insight, such as "the Godbillon-Vey invariant measures the helical wobble of a foliation", that remained mysterious to most mathematicans who read them. This created a high entry barrier: I think many graduate students and mathematicians were discouraged that it was hard to learn and understand the proofs of key theorems.

Second is the issue of what is in it for other people in the subfield. When I started working on foliations, I had the conception that what people wanted was to know the answers. I thought that what they sought was a collection of powerful proven theorems that might be applied to answer further mathematical questions. But that's only one part of the story. More than the knowledge, people want personal understanding. And in our credit-driven system, they also want and need theorem-credits.

I'll skip ahead a few years, to the subject that Jaffe and Quinn alluded to, when I began studying 3-dimensional manifolds and their relationship to hyperbolic geometry. (Again, it matters little if you know what this is about.) I gradually built up over a number of years a certain intuition for hyperbolic three-manifolds, with a repertoire of constructions, examples and proofs. (This process actually started when I was an undergraduate, and was strongly bolstered by applications to foliations.) After a while, I conjectured or speculated that all three-manifolds have a certain geometric structure; this conjecture eventually became known as the geometrization conjecture. About two or three years later, I proved the geometrization theorem for Haken manifolds. It was a hard theorem, and I spent a tremendous amount of effort thinking about it. When I completed the proof, I spent a lot more effort checking the proof, searching for difficulties and testing it against independent information.

I'd like to spell out more what I mean when I say I proved this theorem. It meant that I had a clear and complete flow of ideas, including details, that withstood a great deal of scrutiny by myself and by others. Mathematicians have many different styles of thought. My style is not one of making broad sweeping but careless generalities, which are merely hints or inspirations: I make clear mental models, and I think things through. My proofs have turned out to be quite reliable. I have not had trouble backing up claims or producing details for things I have proven. I am good in detecting flaws in my own reasoning as well as in the reasoning of others.

However, there is sometimes a huge expansion factor in translating from the encoding in my own thinking to something that can be conveyed to someone else. My mathematical education was rather independent and idiosyncratic, where for a number of years I learned things on my own, developing personal mental models for how to think about mathematics. This has often been a big advantage for me in thinking about mathematics, because it's easy to pick up later the standard mental models shared by groups of mathematicians. This means that some concepts that I use freely and naturally in my personal thinking are foreign to most mathematicians I talk to. My personal mental models and structures are 
similar in character to the kinds of models groups of mathematicians sharebut they are often different models. At the time of the formulation of the geometrization conjecture, my understanding of hyperbolic geometry was a good example. A random continuing example is an understanding of finite topological spaces, an oddball topic that can lend good insight to a variety of questions but that is generally not worth developing in any one case because there are standard circumlocutions that avoid it.

Neither the geometrization conjecture nor its proof for Haken manifolds was in the path of any group of mathematicians at the time-it went against the trends in topology for the preceding 30 years, and it took people by surprise. To most topologists at the time, hyperbolic geometry was an arcane side branch of mathematics, although there were other groups of mathematicians such as differential geometers who did understand it from certain points of view. It took topologists a while just to understand what the geometrization conjecture meant, what it was good for, and why it was relevant.

At the same time, I started writing notes on the geometry and topology of 3manifolds, in conjunction with the graduate course I was teaching. I distributed them to a few people, and before long many others from around the world were writing for copies. The mailing list grew to about 1200 people to whom I was sending notes every couple of months. I tried to communicate my real thoughts in these notes. People ran many seminars based on my notes, and I got lots of feedback. Overwhelmingly, the feedback ran something like "Your notes are really inspiring and beautiful, but I have to tell you that we spent 3 weeks in our seminar working out the details of $\S n . n$. More explanation would sure help."

I also gave many presentations to groups of mathematicians about the ideas of studying 3-manifolds from the point of view of geometry, and about the proof of the geometrization conjecture for Haken manifolds. At the beginning, this subject was foreign to almost everyone. It was hard to communicatethe infrastructure was in my head, not in the mathematical community. There were several mathematical theories that fed into the cluster of ideas: threemanifold topology, Kleinian groups, dynamical systems, geometric topology, discrete subgroups of Lie groups, foliations, Teichmüller spaces, pseudo-Anosov diffeomorphisms, geometric group theory, as well as hyperbolic geometry.

We held an AMS summer workshop at Bowdoin in 1980, where many mathematicans in the subfields of low-dimensional topology, dynamical systems and Kleinian groups came.

It was an interesting experience exchanging cultures. It became dramatically clear how much proofs depend on the audience. We prove things in a social context and address them to a certain audience. Parts of this proof I could communicate in two minutes to the topologists, but the analysts would need an hour lecture before they would begin to understand it. Similarly, there were some things that could be said in two minutes to the analysts that would take an hour before the topologists would begin to get it. And there were many other parts of the proof which should take two minutes in the abstract, but that none of the audience at the time had the mental infrastructure to get in less than an hour.

At that time, there was practically no infrastructure and practically no context for this theorem, so the expansion from how an idea was keyed in my head to 
what I had to say to get it across, not to mention how much energy the audience had to devote to understand it, was very dramatic.

In reaction to my experience with foliations and in response to social pressures, I concentrated most of my attention on developing and presenting the infrastructure in what I wrote and in what I talked to people about. I explained the details to the few people who were "up" for it. I wrote some papers giving the substantive parts of the proof of the geometrization theorem for Haken manifolds-for these papers, I got almost no feedback. Similarly, few people actually worked through the harder and deeper sections of my notes until much later.

The result has been that now quite a number of mathematicians have what was dramatically lacking in the beginning: a working understanding of the concepts and the infrastructure that are natural for this subject. There has been and there continues to be a great deal of thriving mathematical activity. By concentrating on building the infrastructure and explaining and publishing definitions and ways of thinking but being slow in stating or in publishing proofs of all the "theorems" I knew how to prove, I left room for many other people to pick up credit. There has been room for people to discover and publish other proofs of the geometrization theorem. These proofs helped develop mathematical concepts which are quite interesting in themselves, and lead to further mathematics.

What mathematicians most wanted and needed from me was to learn my ways of thinking, and not in fact to learn my proof of the geometrization conjecture for Haken manifolds. It is unlikely that the proof of the general geometrization conjecture will consist of pushing the same proof further.

A further issue is that people sometimes need or want an accepted and validated result not in order to learn it, but so that they can quote it and rely on it.

Mathematicians were actually very quick to accept my proof, and to start quoting it and using it based on what documentation there was, based on their experience and belief in me, and based on acceptance by opinions of experts with whom I spent a lot of time communicating the proof. The theorem now is documented, through published sources authored by me and by others, so most people feel secure in quoting it; people in the field certainly have not challenged me about its validity, or expressed to me a need for details that are not available.

Not all proofs have an identical role in the logical scaffolding we are building for mathematics. This particular proof probably has only temporary logical value, although it has a high motivational value in helping support a certain vision for the structure of 3-manifolds. The full geometrization conjecture is still a conjecture. It has been proven for many cases, and is supported by a great deal of computer evidence as well, but it has not been proven in generality. I am convinced that the general proof will be discovered; I hope before too many more years. At that point, proofs of special cases are likely to become obsolete.

Meanwhile, people who want to use the geometric technology are better off to start off with the assumption "Let $M^{3}$ be a manifold that admits a geometric decomposition," since this is more general than "Let $M^{3}$ be a Haken manifold." People who don't want to use the technology or who are suspicious of 
it can avoid it. Even when a theorem about Haken manifolds can be proven using geometric techniques, there is a high value in finding purely topological techniques to prove it.

In this episode (which still continues) I think I have managed to avoid the two worst possible outcomes: either for me not to let on that I discovered what I discovered and proved what I proved, keeping it to myself (perhaps with the hope of proving the Poincaré conjecture), or for me to present an unassailable and hard-to-learn theory with no practitioners to keep it alive and to make it grow.

I can easily name regrets about my career. I have not published as much as I should. There are a number of mathematical projects in addition to the geometrization theorem for Haken manifolds that I have not delivered well or at all to the mathematical public. When I concentrated more on developing the infrastructure rather than the top-level theorems in the geometric theory of 3-manifolds, I became somewhat disengaged as the subject continued to evolve; and I have not actively or effectively promoted the field or the careers of the excellent people in it. (But some degree of disengagement seems to me an almost inevitable by-product of the mentoring of graduate students and others: in order to really turn genuine research directions over to others, it's necessary to really let go and stop oneself from thinking about them very hard.)

On the other hand, I have been busy and productive, in many different activities. Our system does not create extra time for people like me to spend on writing and research; instead, it inundates us with many requests and opportunities for extra work, and my gut reaction has been to say 'yes' to many of these requests and opportunities. I have put a lot of effort into non-credit-producing activities that I value just as I value proving theorems: mathematical politics, revision of my notes into a book with a high standard of communication, exploration of computing in mathematics, mathematical education, development of new forms for communication of mathematics through the Geometry Center (such as our first experiment, the "Not Knot" video), directing MSRI, etc.

I think that what I have done has not maximized my "credits". I have been in a position not to feel a strong need to compete for more credits. Indeed, I began to feel strong challenges from other things besides proving new theorems.

I do think that my actions have done well in stimulating mathematics. 